\title{
Application of advanced biomechanical methods in studying low back pain - recent development in estimation of lower back loads and large-array surface electromyography and findings
}

\author{
This article was published in the following Dove Press journal: \\ Journal of Pain Research \\ 17 July 2017 \\ Number of times this article has been viewed
}

\author{
Babak Bazrgari' \\ Ting $\mathrm{Xia}^{2}$ \\ 'F. Joseph Halcomb III, M.D. \\ Department of Biomedical \\ Engineering, University of Kentucky, \\ Lexington, KY, ${ }^{2} \mathrm{Palmer}$ Center for \\ Chiropractic Research, Palmer \\ College of Chiropractic, Davenport, \\ IA, USA
}

\begin{abstract}
Low back pain (LBP) is a major public health problem and the leading disabling musculoskeletal disorder globally. A number of biomechanical methods using kinematic, kinetic and/or neuromuscular approaches have been used to study LBP. In this narrative review, we report recent developments in two biomechanical methods: estimation of lower back loads and large-array surface electromyography (LA-SEMG) and the findings associated with LBP. The ability to estimate lower back loads is very important for the prevention and the management of work-related low back injuries based on the mechanical loading model as one category of LBP classification. The methods used for estimation of lower back loads vary from simple rigid link-segment models to sophisticated, optimization-based finite element models. In general, reviewed reports of differences in mechanical loads experienced in lower back tissues between patients with LBP and asymptomatic individuals are not consistent. Such lack of consistency is primarily due to differences in activities under which lower back mechanical loads were investigated as well as heterogeneity of patient populations. The ability to examine trunk neuromuscular behavior is particularly relevant to the motor control model, another category of LBP classification. LA-SEMG not only is noninvasive but also provides spatial resolution within and across muscle groups. Studies using LA-SEMG showed that healthy individuals exhibit highly organized, symmetric back muscle activity patterns, suggesting an orderly recruitment of muscle fibers. In contrast, back muscle activity patterns in LBP patients are asymmetric or multifocal, suggesting lack of orderly muscle recruitment. LA-SEMG was also shown capable of capturing unique back muscle response to manual therapy. In conclusion, estimation of low back load and LA-SEMG techniques demonstrated promising potentials for understanding LBP and treatment effects. Future studies are warranted to fully establish clinical validity of these two biomechanical methods.
\end{abstract}

Keywords: low back pain, low back loads, large-array electromyography, narrative review

\section{Introduction}

Despite extensive efforts, low back pain (LBP) persists as a major public health problem and the leading disabling musculoskeletal disorder globally. ${ }^{1-4}$ A recent survey on US health care spending from 1996 to 2013 ranked low back and neck pain as the third costliest disorder at $\$ 87.6$ billion per year, following only diabetes ( $\$ 101.4$ billion) and ischemic heart diseases ( $\$ 88.1$ billion). ${ }^{5}$ In addition, the indirect costs associated with LBP, including loss of employment and household productivity, have been estimated to be up to eight times the direct health care spending in the USA. ${ }^{6}$ It is noteworthy that
Correspondence: Ting Xia

Palmer Center for Chiropractic Research,

Palmer College of Chiropractic, 74I

Brady Street, Davenport, IA 52803, USA

$\mathrm{Tel}+|563884516|$

Email ting.xia@palmer.edu 
while most LBP episodes resolve spontaneously or with some form of treatment in a few weeks, a subgroup of patients ends up developing chronic LBP; ${ }^{7,8}$ a condition, however, accounts for most of the LBP-related suffering and costs. ${ }^{9-15}$ In an analysis of 202,588 insurance claims in the USA, compared to the matched non-chronic LBP patients, chronic LBP patients demonstrated greater comorbidities (e.g., musculoskeletal, neuropathic, and psychological conditions), greater pain medication usage, greater medical resource utilization, and greater direct medical costs. ${ }^{9}$ In European countries, it was found that chronic LBP was responsible for up to $90 \%$ of all expenses. ${ }^{16,17}$

Historically, a number of models for the diagnosis and classification of LBP and associated assessment methods have been proposed. ${ }^{18}$ Particularly relevant to the topic of this review, the mechanical loading model is based on the evidence that mechanical risk factors, such as awkward posture, high loading manual tasks, and exposure to vibration and specific sporting activities, can lead to high spinal load and are usually reported to be associated with the initial low back injury, recurrence of LBP, and eventual development to chronic LBP. ${ }^{19}$ Also relevant is the motor control model that deals with LBP-related impairment in neuromuscular control. It is believed that movement and motor control impairments occur secondary to the presence of pain (e.g., adaptive or protective trunk neuromuscular behavior in response to LBP). ${ }^{20-22}$ Psychological factors such as stress, fear, and anxiety are also known to disrupt motor behavior. ${ }^{23}$ It is noteworthy that these two models are interconnected as pain-related maladaptation in movement, and motor control impairments may lead to abnormal loads in the lower back tissues, which in turn provokes pain. ${ }^{18,24,25}$ Such vicious cycle may be broken if targeted interventions, including exercise, manual therapy, and/or ergonomic design, can be administered to correct the altered trunk neuromuscular behavior (vice versa, to reduce spinal load and/or pain), subsequently leads to reduction in loads experienced in the lower back tissue and ultimately ameliorate pain (vice versa, recovery in trunk neuromuscular behavior).

The ability of biomechanical methods in capturing altered movement and trunk neuromuscular behavior in LBP patients is well documented. Kinematic methods, such as postural sway, lumbar range of motion, and lumbopelvic rhythm, in studying LBP have been systematically reviewed recently by Laird et al. ${ }^{26}$ In addition, Gombatto et $\mathrm{al}^{27}$ systematically reviewed lumbar kinematic characteristics in LBP patients during walking. Electromyography (EMG)based methods, such as surface EMG (SEMG), in studying altered trunk neuromuscular behavior in LBP patients have been thoroughly examined by van Dieën et $\mathrm{al}^{22}$ and Geisser et al. ${ }^{28}$ The findings in EMG have led to the new theory of secondary, pain-related trunk neuromuscular adaptation over the original belief of primary neuromuscular impairment in LBP patients. ${ }^{21-23}$ In addition, Ghamkhar and Kahlaee ${ }^{29}$ systematically reviewed activation pattern of trunk muscles in LBP patients during walking. Kinetic methods have also been applied to study LBP, although are not as popular as kinematic and EMG methods. It is noteworthy that some pathological conditions, particularly those associated with the spinal structures, may also lead to abnormal load in spinal tissues and pain. These conditions include, but not limited to, degenerated disk and facets, herniated disk, spondylolisthesis, spinal stenosis, and tumor. Both standard and advanced medical imaging techniques are available for diagnosing these conditions and a better classification of LBP. ${ }^{30}$

In this narrative review, we report recent developments in applying kinetic methods to estimate lower back loads in LBP patients and asymptomatic individuals when performing a variety of physical tasks. In addition, we report a novel EMG technique using a large array of surface sensors (large-array surface electromyography [LA-SEMG]) and its application in obtaining valuable spatial information of back muscle activity patterns in LBP patients.

\section{Kinetic methods in estimating lower back loads}

There is extensive evidence suggesting that mechanical loading in the human lower back can directly or indirectly irritate pain-sensitive nerve endings in the lower back tissues and cause LBP. $^{19,31,32}$ Therefore, over the past several decades, study of mechanical loads in the human lower back has been the focus of many research efforts that were aimed at understanding the underlying mechanism(s) linking exposure to LBP risk factors and LBP occurrence and recurrence. Mechanical loads experienced in the lower back tissues are directly affected by spinal equilibrium and stability (SEAS). Spinal equilibrium is a delicate balance between the physical demands of an activity and the active and passive responses of lower back tissues attached to the spine. Spinal stability, however, is the capacity of lower back tissues to regulate and sustain spine equilibrium within an optimal range that provides the spine both its rigidity and flexibility under diverse conditions. Mechanical loads experienced in lower back tissues, as shown in Figure 1, are the result of the active and passive mechanical responses of lower back tissues to physical demand to assure SEAS. More specifically, active (motor or sensory) tissue responses to physical 


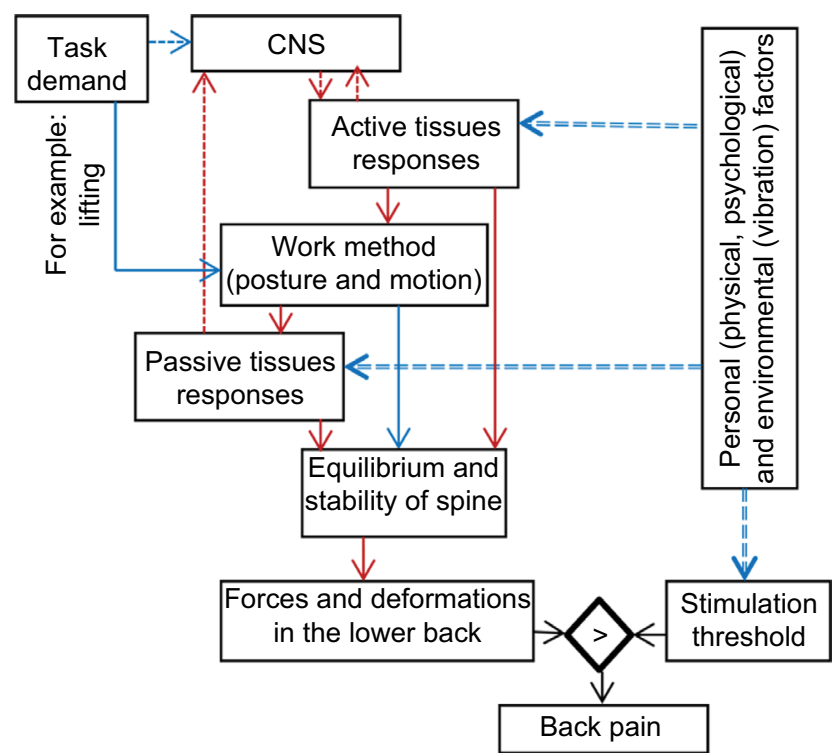

Figure I A simplified mechanical pathway linking exposure to LBP risk factors with the development of LBP.

Notes: Arrows indicate mechanical (solid line), information (thin broken line), and other types of interactions (thick broken line). Blue and red colors represent external and internal variables, respectively.

Abbreviations: LBP, low back pain; CNS, central nervous system.

demands of activity, which contribute to SEAS, determine the behavioral methods used (e.g., lumbar posture and trunk motion during walking). Resultant postures and motions of the lower back, in turn, determine the passive tissue contributions (due to deformation) to SEAS.

Two general mechanisms involving lower back mechanics have been mainly investigated in search of potential pathways linking exposure to LBP risk factors to LBP. ${ }^{33,34}$ These two mechanisms include tissue failure or nerve irritation due to 1) an instantaneous experience of excessive mechanical loads in the lower back tissues that exceed threshold of tissue failure or nerve excitation or 2) cumulative increase in loads experienced in the lower back tissues as a result of timedependent change in active (e.g., muscle fatigue) and passive (e.g., creep deformation) behaviors of lower back tissues such that exceed a decreasing threshold of tissue failure (e.g., due to accumulation of microdamage) or nerve excitation. While there is evidence in support of association between both cumulative $\mathrm{e}^{35-37}$ and peak instantaneous $\mathrm{s}^{37,38}$ loads in the lower back tissues and occurrence of LBP, it is not clear if these two pathways may have also a role in the progression of an acute LBP condition to chronic/recurrent LBP or in linking LBP treatments with LBP recovery. As a first step toward answering such a research gap, we have reviewed reports of differences in lower back loads between patients with LBP (both acute and chronic) and asymptomatic individuals when performing a variety of physical tasks.
To evaluate how the presence of LBP influences loads in the lower back during lifting tasks, Marras et $\mathrm{al}^{39}$ performed a study wherein lower back loads were evaluated in 22 patients with LBP (both acute and chronic) and 22 asymptomatic controls when they completed two tasks. The investigators used an EMG-assisted model to evaluate lower back loads by estimating the net moment, compression and shear forces at the L5/S1 level of the spine. In this method, the mechanical demand of task at the L5/S1 level (i.e., net external moment) that should be balanced internally by lower back tissues is calculated first. Such mechanical demand is then used along with measured activity of select trunk muscles to estimate balancing forces provided by 10 muscles crossing the L5/S1 level. Under a restrained lifting task, designed to impose a similar trunk-flexed posture, net moment at the L5/S1 level was found to be larger ( 12 Nm in average) in patients compared to controls. To account for differences in participants' body weight, compression and lateral shear force at the L5/S1 level were normalized to the net moment before comparison between groups. Such normalized compression and lateral shear force at the L5/ S1 level were also found to be, respectively, $26 \%$ and $75 \%$ higher in patients. The observed differences in spinal loads under restrained lifting tasks were suggested to be primarily due to differences in muscle contribution to SEAS, which involved more coactivation in patients. Similar differences (18 Nm in average) in net moment were found under a free lifting task between patients and controls, but no differences in normalized compression and shear forces were found between the groups. The authors suggested that spinal loads were decreased by patients using a kinematics compensation strategy when performing the free lifting tasks.

To verify if patients with chronic LBP would use a lifting strategy that decreases lower back loads, Larivière et $\mathrm{al}^{40}$ evaluated lower back loads in 15 patients with chronic LBP and 18 controls when they performed both symmetric and asymmetric lifting and lowering of a $12 \mathrm{~kg}$ box. Rigid linksegment models of whole body were used to estimate threedimensional net external moments at the L5/S1 level using both bottom-up (i.e., starting calculation from the ground and ending at the L5/S1 level) and top-down (i.e., starting calculation from the hands and ending at the L5/S1 level) approaches. The investigators used a polynomial function of the L5/S1 moment to estimate the compression force at the L5/S1 level. No differences in moment and compression at the L5/S1 level were observed between the two groups.

To further explore how presence of LBP influences loads in the lower back, Marras et $\mathrm{al}^{41}$ reported another study 
that in addition to symmetric lifting involved asymmetric lifting exertion. The study involved 62 patients with mean (SD) duration of 10.2 months (13.6 months) of LBP and 61 asymptomatic controls. Participants performed several lifting tasks that were different in terms of distance to subject (near versus far), height of lifting origin (knee, waist, and shoulder heights $)$, asymmetry $\left(0^{\circ}, 45^{\circ}\right.$, and $90^{\circ}$ clockwise and $45^{\circ}$ and $90^{\circ}$ counter clockwise, all with respect to the sagittal plane), and load (4.5, 6.8, 9.1, and $11.4 \mathrm{~kg})$. Using their EMGassisted model, the investigators estimated compression and shear forces at the L5/S1 level. Larger compression and shear forces were found in patients versus controls. Furthermore, lower back loads were found to depend on lifting origin and to a lesser extent on the magnitude of the lifted load. The authors suggested that subjective perception of the need for spinal stability might have driven higher coactivation of trunk muscles in patients that resulted in larger compression and shear forces. It should, however, be noted that patients in this study were in average $\sim 14 \mathrm{~kg}$ heavier than controls and reported results were not normalized to account for the effects of body weight.

Motivated by scarcity of information related to lower back loads in patients with LBP, Shum et $\mathrm{al}^{42,43}$ conducted a couple of studies to investigate lower back loads in patients with LBP when they performed trunk forward bending and backward return as well as sit-to-stand and stand-to-sit activities. They studied 40 patients with acute LBP ( $<12$ weeks duration) and 20 asymptomatic controls. Among patients with LBP, 20 of them had a positive sign of straight leg raise. Three-dimensional net moments at the L5/S1 level were calculated as the measure of lower back loads. This was done using a rigid body link-segment model of human body from feet to the L5/S1 level. For trunk forward bending and backward return, the net moment at the L5/S1 level was smaller in patients at the end range of trunk forward bending but was larger at smaller bending angles (i.e., $15^{\circ}$, $30^{\circ}$, and $45^{\circ}$ ). Such differences in net moment at the L5/S1 level between patients and asymptomatic individuals were found to be more pronounced in patients with a positive sign of single raise leg. For sit-to-stand and stand-to-sit activities, the net moment at the L5/S1 level was smaller in the main plane of movement (the sagittal plane) but larger in frontal and transverse planes among patients with LBP compared to asymptomatic controls.

Differences in lower back loads between patients with LBP and control have also been reported for unstable sitting conditions. Freddolini et $\mathrm{al}^{44}$ used a two-dimensional (2D; planar) model of a seated person to calculate the net moment at the L5/S1 level when participants hold a seated posture that was unstable only in the sagittal plane. The study included 31 asymptomatic individuals and 23 patients with LBP (duration $>6$ weeks). No difference was found in the net moment at the L5/S1 level between patients and controls. In another study, Shahvarpour et $\mathrm{al}^{45}$ investigated the loads in the lower back under a three dimensionally unstable seated posture to investigate the safety of using a wobble chair in exercises and rehabilitation therapies. The investigators used a kinematicsdriven finite element model (FEM) of the human spine to calculate the trunk muscular response to physical demand of the simulated activity and then to estimate the resultant compression and shear forces acting at each intervertebral disk between the T12 and the S1 spinal levels. Six patients with chronic LBP and six asymptomatic controls tried to hold a balanced seated posture on a wobble chair for $60 \mathrm{~s}$. No differences in spinal loads were found between the groups. Peak compression and anteroposterior and mediolateral shear forces were respectively $1473^{\circ} \mathrm{N}, 691^{\circ} \mathrm{N}$, and $153^{\circ} \mathrm{N}$ in controls and $1720^{\circ} \mathrm{N}, 687^{\circ} \mathrm{N}$, and $208^{\circ} \mathrm{N}$ in patients with chronic LBP. It should be noted, however, that the approach used in the study of Shahvarpour et al did not account for antagonistic co-contraction of trunk muscles, which as suggested by the investigators could have increased the estimated spinal loads, especially in patients with chronic LBP.

We have recently conducted a study to investigate differences in mechanical demand of lowering and lifting a light load (i.e., $4.5 \mathrm{~kg}$ ) in the sagittal plane on the lower back between a group of 19 females with acute LBP and a control group of 19 asymptomatic females. ${ }^{46}$ Rigid linksegment models of the human body from the feet to the L5/S1 level were used to estimate mechanical demand of the task. Mechanical demand of the task included the net moment, axial and shearing components of the reaction force at the L5/S1 level. No differences in the peak net moment at the L5/S1 level were found between the patients and controls; however, the L5/S1 shearing (40-50 years age group) and axial forces at the time of peak net moment were, respectively, larger and smaller in patients vs. controls.

In addition, we have conducted a study investigating spinal load in LBP patients who received manual therapy. ${ }^{47}$ Specifically, 82 patients with chronic LBP received 12 sessions of thrust spinal manipulation over 6 weeks. Spinal load was estimated using an inverse kinetic method developed by Triano and Schultz. ${ }^{48}$ Among three clinicians who delivered the treatment, we found significant differences in lumbar reactive force and moment in patients among the three doctors. ${ }^{49}$ 
Although it has not been yet implemented to assess low back load during physical tasks, it is our opinion that optimization-based FEM (e.g., optimized with kinematic, EMG, and/or force-plate data) is a well-suited approach for such a purpose. Availability of powerful computational resources and advances in image-based geometrically and materially personalized $\mathrm{FEM}^{50,51}$ offers a unique research platform for study of lower back load in patients with LBP, particularly when patients have pathological conditions known to affect low back load. For example, Tsouknidas et a ${ }^{52}$ applied FEM to simulate healthy and osteoporotic lumbosacral spine and found that osteoporosis led to increased facet joint load and even more pronounced with coexistence of degenerated disk. Currently, there is no report considering comorbidity when analyzing low back load in patients with LBP. This gap should be filled in future studies with assistance of FEM.

In summary, among the studies reviewed, three involved patients with acute LBP, ${ }^{42,43,46}$ three involved patients with chronic LBP, ${ }^{40,45,47}$ and three included a mix of patients with acute and chronic LBP. ${ }^{39,41,44}$ The limited number of studies in each patient group makes it difficult to draw any conclusion related to the potential role of lower back mechanical loads in deterioration and/or amelioration of an existing episode of LBP. Therefore, although the role of lower back loads in occurrence of LBP is strongly supported in the current literature, more studies should be conducted in future to help address whether lower back loads also play a role in deterioration and/or amelioration of an existing episode of LBP. In general, reviewed reports of differences in mechanical loads experienced in lower back tissues between patients with LBP and asymptomatic individuals are not consistent. This could in part be due to differences in activities under which lower back loads were estimated. In our recent works, we have observed that patients significantly changed their trunk kinematics when performing a lowering and lifting task as compared to a free-style trunk forward bending and backward return. ${ }^{53,54}$ Specifically, patients vs. controls adopted a much smaller thorax range of rotation in the lowering and lifting task (i.e., 75.2 vs. 85.4) than in free-style forward bending (104.6 vs. 99.1). The reduction in the peak thoracic rotation in patients was achieved by a reduction in the lumbar contribution to the thoracic rotation from $43^{\circ}$ to $32.6^{\circ}$ ( $\sim 24 \%$ reduction), while the reduction in the lumbar contribution to the thoracic rotation in the control group was from $55.7^{\circ}$ to $51.4^{\circ}$ ( $\sim 8 \%$ reduction). Smaller thorax rotation imposes less gravitational demand on the L5/S1 net moment, whereas smaller lumbar flexion reduces passive contribution of lower back tissues in offsetting the
L5/S1 net moment (i.e., increasing the demand on the active muscle contribution).

\section{Large-array surface electromyography}

EMG, including needle/fine-wire EMG (NEMG/FWEMG) and SEMG techniques, has been used extensively to study LBP. While capable of producing muscle-specific and even motor-specific signals, the NEMG/FWEMG approach is invasive and painful to use. On the other hand, the SEMG approach is noninvasive, but suffers from low spatial resolution. To combine the benefits of these two EMG approaches, a novel approach utilizing LA-SEMG was prototyped by Prutchi $^{55}$ in 1995. The author illustrated both the theoretical foundation and the hardware of the system in the work. Additionally, the author tested the system on the biceps muscle during hand lifting, showing promising results for both basic science and clinical applications. Similar to the conventional EMG approaches, techniques to decompose LA-SEMG signals to individual motor unit signals have been made available. ${ }^{56}$ The latest development in LA-SEMG includes the use of a stretchable sensor pad that may significantly expand the application scenarios of the technique..$^{57,58}$

The utilization of LA-SEMG to study LBP was reported shortly after the work of Prutchi ${ }^{55}$ in 1995. In 2003, Finneran et al ${ }^{59}$ applied LA-SEMG to determine if the spatial pattern of back muscle activity in LBP patients differed from healthy controls. Particularly, a 9×7 (63)-channel LA-SEMG system (62 electrodes plus one common ground located at the center of the pad) measuring $25 \mathrm{~cm}$ tall by $19 \mathrm{~cm}$ wide was attached to the low back region bilaterally. Two-dimensional muscle activity scans (root mean square [RMS] value of the voltage over $1 \mathrm{~s}$ ) were taken during three tasks, including upright standing, trunk forward flexion in $20^{\circ}$, and standing while holding a 3-pound dumbbell in each hand. Two reviewers, blinded to group status, qualitatively described the visual characteristics of each scan. The study team recruited 13 participants with acute LBP, 25 participants with chronic LBP, and 163 painfree controls. Additionally, participants with acute LBP were followed up for up to 6 weeks. In the pain-free population, $>90 \%$ participants demonstrated balanced regional muscle activity patterns (nominally identified as diamond, Vee, and columnar) symmetrically over both sides of the spine. In addition, higher muscle activity was observed in muscles located closer to the spinal column and from L3 to S1. In contrast, participants with acute LBP demonstrated increased muscle activity asymmetrically on the painful side, or multifocal, also with higher RMS values on the painful side. Participants with 
chronic LBP showed asymmetrical and/or multifocal patterns of muscle activity similar to participants with acute LBP. In addition, participants with LBP appeared to use different muscle groups than the controls by relying more on muscles more laterally located from the spine. Among the three participants reporting full recovery during the 6-week follow-up period, the scan returned to normal (e.g., symmetric). Of 10 participants not improved, eight continued to have abnormal scans at 6-week follow-up, while two showed a normal pattern. The authors were unable to follow the chronic population over time. Overall, the study findings demonstrated the ability of LA-SEMG to distinguish muscle activity patterns between LBP and pain-free populations.

To further validate the LA-SEMG system used in the study by Finneran et al, ${ }^{59}$ Reger et al ${ }^{60}$ examined back muscle activity in 44 participants with acute LBP and 164 healthy controls with the emphasis to develop a classification method for differentiating LA-SEMG scan obtained from the two groups. The algorithm of the classification model was based on a quadratic discriminant function using demographics, self-reported categorization of health, and LA-SEMG scan. After establishing the model, it was applied to calculate the posterior probability of membership (acute LBP or healthy) for each participant using Bayes' theorem. The same minimal low back stress tasks used in the study by Finneran et al ${ }^{59}$ were performed in this study to obtain LA-SEMG scans. The results indicated LA-SEMG scans obtained during flexion to be most promising in terms of classifying participants into acute LBP or healthy group. Particularly, the model using the flexion data correctly reclassified $95.5 \%$ (42/44) of the acute LBP participants and 99.4\% (160/161) of the healthy participants. The model using the weight holding data and the upright standing data produced good results for classifying healthy participants, but had difficulties classifying participants with acute LBP, especially with the upright standing data. It is likely that the lower muscle activity in these two tasks contributed to the reduced classification accuracy in participants with acute LBP. Overall, the findings from the study supported the clinical validity of the proposed classification methodology based on LA-SEMG.

$\mathrm{Hu}$ et $\mathrm{al}^{61}$ proposed a more advanced, dynamic LA-SEMG topology method (a series of scans during a dynamic task) to examine muscle activity patterns in participants with chronic LBP and the effects of rehabilitation. In all, 20 healthy participants and 15 chronic LBP participants were recruited in the study. A $7 \times 3$ LA-SEMG system (16 active electrodes, three reference electrodes, and two ground electrodes) was attached bilaterally over the low back muscles from L2 to L5, and scans were obtained when participants were performing lumbar flexion-extension for up to $30^{\circ}$ in flexion before returning to the upright stand posture. Participants with chronic LBP further underwent 12 weeks of physical therapy, and their posttreatment LA-SEMG scans were obtained. To visualize dynamic changes in LA-SEMG, the RMS values of voltage signals at each active electrode were calculated as a function of both position and time. A linear cubic spline interpolation was then applied to create a 2D topographic image from the 16 active electrodes at each time point (a frame of scan). In addition to qualitative visual inspection of the scans, four quantitative parameters, including relative area (RA), relative width (RW), relative height (RH), and width-to-height ratio $(\mathrm{W} / \mathrm{H})$, were used to measure topographic features. Visual inspection of the scans obtained from healthy controls (18/20) typically demonstrated a symmetric pattern throughout the flexion-extension motion with a high activity concentrated in the middle and lower regions. In contrast, LBP participants demonstrated a broader and more disorganized, or an asymmetric, distribution of high activity than controls. Posttreatment LA-SEMG scans from LBP participants showed recovery in the activity pattern toward normal. Quantitative analysis demonstrated significant differences in RA and $\mathrm{W} / \mathrm{H}$ between the two groups. Treatment led to recovery in RA and $\mathrm{W} / \mathrm{H}$ toward normal values. No difference in RW and RH was observed between groups or pre-post treatment.

Other forms of physical tasks and LA-SEMG scan pattern recognition techniques have been used to distinguish between LBP and healthy populations. Abboud et al ${ }^{62}$ applied dispersion analyses to characterize trunk motor variability from LA-SEMG scans obtained in patients with chronic LBP and healthy controls who underwent a modified Sørensen endurance test. Particularly, activity of right and left erector spinae was recorded using two 64-electrode LA-SEMG pads centered at L3. The center of gravity of $0.5 \mathrm{~s}-\mathrm{RMS}$ values on each scan was determined using dispersion analyses. The modified Sørensen test consisted of isometric back extension at $30 \%$ of maximum voluntary contraction until exhaustion. The endurance time of the fatigue task was divided into six equal segments. The traveling of the center of gravity during each segment was used to quantify global migration of muscular activity (i.e., trunk motor variability). The authors found that trunk motor variability was higher in healthy controls than that in participants with LBP. Additionally, trunk motor variability increased with the development of muscle fatigue in participants with LBP, but with a lower increase on the left side when compared to healthy controls. 
LA-SEMG can also be useful to examine the effects of passive physical stimulation, such as manual therapy, on LBP. Pagé et $\mathrm{a}^{63}$ applied simulated spinal manipulation of four different levels of force on 26 participants with chronic LBP and 25 gender-matched healthy controls. The simulated spinal manipulation was delivered using a programmable indenter instrument over the $\mathrm{L} 3$ spinous process. Back muscle response was recorded bilaterally at L3 using two $8 \times 8$ (64) electrode LA-SEMG pads. The RMS value was computed for each electrode during the thrust phase of spinal manipulation (0-50 ms time window and 50-100 ms time window). The authors found a dose-response relationship between force and neuromuscular response. In addition, a higher stimulation force led to an early initiation of muscle response during the " $0-50 \mathrm{~ms}$ time window" and was maintained through the " $50-100 \mathrm{~ms}$ time window". Further spatial analysis results revealed that the neuromuscular response amplitude decreased as the distance from the thrust point increased in a concentric pattern.

In summary, among the five studies reviewed, two involved patients with acute $\mathrm{LBP}^{59,60}$ four involved patients with chronic LBP, ${ }^{59,61-63}$ and two involved treatment with one on patients with acute LBP and one on patients with chronic LBP. $^{59,63}$ Although most findings are semiquantitative or qualitative, these studies clearly demonstrated the capability of LA-SEMG in providing spatial information of back muscle activity patterns in LBP patients. Most importantly, these study results revealed that healthy individuals exhibit highly organized, symmetric back muscle activity patterns with maximum activity closer to the spine and from S1 to L3. Such an orderly recruitment from muscle fibers located closer to the spine and at the lower lumbar levels and then muscle fibers away from the spine and at upper levels indicates an optimized spine stabilization strategy in response to increased spinal load and demand for stability at lower lumbar levels. In contrast, back muscle activity patterns in LBP patients are asymmetric or multifocal, suggesting the lack of orderly muscle recruitment and less optimized stabilization strategy. In addition, LA-SEMG was also shown capable of capturing acute back muscle response to manual therapy, as well as cumulative effects on muscle recruitment patterns over a period of treatment. Therefore, even with limited evidence, LA-SEMG demonstrates promising potential in studying LBP and treatment effects. Future studies are warranted to fully establish clinical validity of LA-SEMG.

\section{Acknowledgments}

The role of corresponding author is not an indication of seniority of authorship.

\section{Author contributions}

All authors contributed toward literature search and review, drafting and revising the paper and agree to be accountable for all aspects of the work.

\section{Disclosure}

The authors report no conflicts of interest in this work.

\section{References}

1. Breivik H, Collett B, Ventafridda V, Cohen R, Gallacher D. Survey of chronic pain in Europe: prevalence, impact on daily life, and treatment. Eur J Pain. 2006;10(4):287.

2. Pransky G, Borkan JM, Young AE, Cherkin DC. Are we making progress? The tenth international forum for primary care research on low back pain. Spine (Phila Pa 1976). 2011;36(19):1608-1614.

3. Soni A. Top 10 Most Costly Conditions among Men and Women, 2008 Estimates for the US Civilian Noninstitutionalized Adult Population, Age 18 and Older. Rockville, MD: Agency for Healthcare Research and Quality; 2011.

4. Vos T, Flaxman AD, Naghavi M, et al. Years lived with disability (YLDs) for 1160 sequelae of 289 diseases and injuries 1990-2010: a systematic analysis for the global burden of disease study 2010. Lancet. 2012;380(9859):2163-2196.

5. Dieleman JL, Baral R, Birger M, et al. US spending on personal health care and public health, 1996-2013. JAMA. 2016;316(24):2627-2646.

6. Dagenais S, Caro J, Haldeman S. A systematic review of low back pain cost of illness studies in the united states and internationally. Spine J. 2008;8(1):8-20.

7. Van Tulder M, Becker A, Bekkering T, et al; COST B13 Working Group on Guidelines for the Management of Acute Low Back Pain in Primary Care. Chapter 3 European guidelines for the management of acute nonspecific low back pain in primary care. Eur Spine J. 2006;15(Suppl 2): s169-s191.

8. Elfering A, Mannion AF. Epidemiology and Risk Factors of Spinal Disorders. Spinal Disorders. Berlin: Springer; 2008:153-173.

9. Gore M, Sadosky A, Stacey BR, Tai KS, Leslie D. The burden of chronic low back pain: clinical comorbidities, treatment patterns, and health care costs in usual care settings. Spine. 2012;37(11):E668-E677.

10. Andersson GB. Epidemiological features of chronic low-back pain. Lancet. 1999;354(9178):581-585.

11. Ekman M, Jönhagen S, Hunsche E, Jönsson L. Burden of illness of chronic low back pain in Sweden: a cross-sectional, retrospective study in primary care setting. Spine. 2005;30(15):1777-1785.

12. Frank AO, De Souza LH, McAuley JH, Sharma V, Main CJ. A crosssectional survey of the clinical and psychological features of low back pain and consequent work handicap: use of the Quebec task force classification. Int J Clin Pract. 2000;54(10):639-644.

13. Hong J, Reed C, Novick D, Happich M. Costs associated with treatment of chronic low back pain: an analysis of the UK general practice research database. Spine. 2013;38(1):75-82.

14. Hoy D, Bain C, Williams G, et al. A systematic review of the global prevalence of low back pain. Arthritis Rheum. 2012;64(6):2028-2037.

15. Gjesdal S, Bratberg E, Maeland JG. Musculoskeletal impairments in the Norwegian working population: the prognostic role of diagnoses and socioeconomic status: a prospective study of sickness absence and transition to disability pension. Spine. 2009;34(14):1519-1525.

16. Juniper M, Le TK, Mladsi D. The epidemiology, economic burden, and pharmacological treatment of chronic low back pain in France, Germany, Italy, Spain and the UK: a literature-based review. Expert Opin Pharmacother. 2009;10(16):2581-2592.

17. Wenig CM, Schmidt CO, Kohlmann T, Schweikert B. Costs of back pain in Germany. Eur J Pain. 2009;13(3):280-286.

18. O'Sullivan P. Diagnosis and classification of chronic low back pain disorders: maladaptive movement and motor control impairments as underlying mechanism. Man Ther. 2005;10(4):242-255. 
19. Adams MA, Bogduk N, Burton K, Dolan P. The Biomechanics of Back Pain. 2nd ed. London: Churchill Livingstone; 2006.

20. Hodges PW. Pain and motor control: from the laboratory to rehabilitation. J Electromyogr Kinesiol. 2011;21(2):220-228.

21. Hodges PW, Tucker K. Moving differently in pain: a new theory to explain the adaptation to pain. Pain. 2011;152(3 suppl):S90-S98.

22. van Dieën JH, Selen LP, Cholewicki J. Trunk muscle activation in lowback pain patients, an analysis of the literature. J Electromyogr Kinesiol. 2003;13(4):333-351.

23. Hodges PW, Moseley GL. Pain and motor control of the lumbopelvic region: effect and possible mechanisms. J Electromyogr Kinesiol. 2003;13(4):361-370.

24. Burnett AF, Cornelius MW, Dankaerts W, O'sullivan PB. Spinal kinematics and trunk muscle activity in cyclists: a comparison between healthy controls and non-specific chronic low back pain subjects-a pilot investigation. Man Ther. 2004;9(4):211-219.

25. Dankaerts W, O'Sullivan P, Burnett A, Straker L. Differences in sitting postures are associated with nonspecific chronic low back pain disorders when patients are subclassified. Spine. 2006;31(6):698-704.

26. Laird RA, Gilbert J, Kent P, Keating JL. Comparing lumbo-pelvic kinematics in people with and without back pain: a systematic review and meta-analysis. BMC Musculoskelet Disord. 2014;15:229.

27. Gombatto SP, Brock T, DeLork A, Jones G, Madden E, Rinere C. Lumbar spine kinematics during walking in people with and people without low back pain. Gait Posture. 2015;42(4):539-544.

28. Geisser ME, Ranavaya M, Haig AJ, et al. A meta-analytic review of surface electromyography among persons with low back pain and normal, healthy controls. J Pain. 2005;6(11):711-726.

29. Ghamkhar L, Kahlaee AH. Trunk muscles activation pattern during walking in subjects with and without chronic low back pain: a systematic review. PM R. 2015;7(5):519-526.

30. Jarvik JG, Deyo RA. Diagnostic evaluation of low back pain with emphasis on imaging. Ann Intern Med. 2002;137(7):586-597.

31. Bogduk N. Clinical Anatomy of the Lumbar Spine and Sacrum. London: Churchill Livingstone; 2005.

32. Marras WS. The Working Back: A Systems View. Hoboken, NJ: John Wiley \& Sons; 2008.

33. Chaffin DB. The evolving role of biomechanics in prevention of overexertion injuries. Ergonomics. 2009;52(1):3-14.

34. Marras WS. The complex spine: the multidimensional system of causal pathways for low-back disorders. Hum Factors. 2012;54(6):881-889.

35. Coenen P, Kingma I, Boot CR, Twisk JW, Bongers PM, van Dieën JH. Cumulative low back load at work as a risk factor of low back pain: a prospective cohort study. J Occup Rehabil. 2013;23(1):11-18.

36. Kumar S. Cumulative load as a risk factor for back pain. Spine. 1990;15(12):1311-1316.

37. Norman R, Wells R, Neumann P, Frank J, Shannon H, Kerr M. A comparison of peak vs cumulative physical work exposure risk factors for the reporting of low back pain in the automotive industry. Clin Biomech. 1998;13(8):561-573.

38. Neumann W, Wells R, Norman RW, et al. A posture and load sampling approach to determining low-back pain risk in occupational settings. Int J Ind Ergon. 2001;27(2):65-77.

39. Marras WS, Davis KG, Ferguson SA, Lucas BR, Gupta P. Spine loading characteristics of patients with low back pain compared with asymptomatic individuals. Spine. 2001;26(23):2566-2574.

40. Larivière C, Gagnon D, Loisel P. A biomechanical comparison of lifting techniques between subjects with and without chronic low back pain during freestyle lifting and lowering tasks. Clin Biomech. 2002; 17(2):89-98.

41. Marras WS, Ferguson SA, Burr D, Davis KG, Gupta P. Spine loading in patients with low back pain during asymmetric lifting exertions. Spine J. 2004;4(1):64-75.

42. Shum GL, Crosbie J, Lee RY. Three-dimensional kinetics of the lumbar spine and hips in low back pain patients during sit-to-stand and standto-sit. Spine. 2007;32(7):E211-E219.
43. Shum GL, Crosbie J, Lee RY. Back pain is associated with changes in loading pattern throughout forward and backward bending. Spine. 2010;35(25):E1472-E1478.

44. Freddolini M, Strike S, Lee RY. The role of trunk muscles in sitting balance control in people with low back pain. J Electromyogr Kinesiol. 2014;24(6):947-953.

45. Shahvarpour A, Shirazi-Adl A, Larivière C, et al. Active-passive biodynamics of the human trunk when seated on a wobble chair. J Biomech. 2016;49(6):939-945.

46. Shojaei I, Salt E, Hooker Q, Bazrgari B. Mechanical demands on the lower back in patients with acute low back pain during a symmetric lowering and lifting task. $J$ Biomech. In press 2017.

47. Xia T, Wilder DG, Gudavalli MR, et al. Study protocol for patient response to spinal manipulation - a prospective observational clinical trial on physiological and patient-centered outcomes in patients with chronic low back pain. BMC Complement Altern Med. 2014;14:292.

48. Triano J, Schultz AB. Loads transmitted during lumbosacral spinal manipulative therapy. Spine (Phila Pa 1976). 1997;22(17):1955-1964.

49. Xia T, Long C, Gudavalli MR, et al. Towards the understanding of dosage of spinal manipulative therapy - a preliminary study on spine segmental load. 40th Annual Meeting of the American Society of Biomechanics. Raleigh. NC2016.

50. Fagan MJ, Julian S, Mohsen AM. Finite element analysis in spine research. Proc Inst Mech Eng H. 2002;216(5):281-298.

51. Jones AC, Wilcox RK. Finite element analysis of the spine: towards a framework of verification, validation and sensitivity analysis. Med Eng Phys. 2008;30(10):1287-1304.

52. Tsouknidas A, Sarigiannidis SO, Anagnostidis K, Michailidis N, Ahuja $\mathrm{S}$. Assessment of stress patterns on a spinal motion segment in healthy versus osteoporotic bony models with or without disc degeneration: a finite element analysis. Spine J. 2015;15(3):S17-S22.

53. Shojaei I, Salt EG, Hooker Q, Van Dillen LR, Bazrgari B. Comparison of lumbo-pelvic kinematics during trunk forward bending and backward return between patients with acute low back pain and asymptomatic controls. Clin Biomech. 2017;41:66-71.

54. Shojaei I, Vazirian M, Salt EG, Van Dillen LR, Bazrgari B. Timing and magnitude of lumbar spine contribution to trunk forward bending and backward return in patients with acute low back pain. J Biomech. 2017;53:71-77.

55. Prutchi D. A high-resolution large array (HRLA) surface EMG system. Med Eng Phys. 1995;17(6):442-454.

56. Kleine BU, van Dijk JP, Lapatki BG, Zwarts MJ, Stegeman DF. Using two-dimensional spatial information in decomposition of surface EMG signals. J Electromyogr Kinesiol. 2007;17(5):535-548.

57. Kim DH, Ghaffari R, Lu N, Rogers JA. Flexible and stretchable electronics for biointegrated devices. Annu Rev Biomed Eng. 2012;14:113-128.

58. Kim N, Lim T, Song K, Yang S, Lee J. Stretchable multichannel electromyography sensor array covering large area for controlling home electronics with distinguishable signals from multiple muscles. ACS Appl Mater Interfaces. 2016;8(32):21070-21076.

59. Finneran MT, Mazanec D, Marsolais ME, Marsolais EB, Pease WS. Large-array surface electromyography in low back pain: a pilot study. Spine. 2003;28(13):1447-1454.

60. Reger SI, Shah A, Adams TC, et al. Classification of large array surface myoelectric potentials from subjects with and without low back pain. J Electromyogr Kinesiol. 2006;16(4):392-401.

61. Hu Y, Siu SH, Mak JN, Luk KD. Lumbar muscle electromyographic dynamic topography during flexion-extension. J Electromyogr Kinesiol. 2010;20(2):246-255.

62. Abboud J, Nougarou F, Pagé I, Cantin V, Massicotte D, Descarreaux M. Trunk motor variability in patients with non-specific chronic low back pain. Eur J Appl Physiol. 2014;114(12):2645-2654.

63. Pagé I, Nougarou F, Descarreaux M. Neuromuscular response amplitude to mechanical stimulation using large-array surface electromyography in participants with and without chronic low back pain. J Electromyogr Kinesiol. 2016;27:24-29. 
The Journal of Pain Research is an international, peer reviewed, open access, online journal that welcomes laboratory and clinical findings in the fields of pain research and the prevention and management of pain. Original research, reviews, symposium reports, hypothesis formation and commentaries are all considered for publication
The manuscript management system is completely online and includes a very quick and fair peer-review system, which is all easy to use. Visit http://www.dovepress.com/testimonials.php to read real quotes from published authors. 